\title{
14,000 Volt Electrical Injury to Bilateral Upper Extremities: A Case Report
}

\author{
J. Lee, H. Sinno, A. Perkins, Y. Tahiri, \\ M. Luc
}

\begin{abstract}
Electrical burns are among the most devastating of burn injuries. High voltage electrical injuries result in extensive deep tissue damage and are associated with multiple complications, long term morbidity, and a high mortality rate. We describe the case of a 47 year-old electric company linesman who suffered a high voltage electrical injury (HVEI) of 14,000 volts to bilateral hands and wrists managed by the Division of Plastic and Reconstructive Surgery at the McGill University Health Center in Montreal, Quebec, Canada. His management included multiple operative procedures, including escharotomies, fasciotomies, serial debridements, and bilateral pedicle groin flaps, and amputation of his left hand.
\end{abstract}

KEYWORDS: High-voltage, Electrical Injury, Escharotomy, Fasciotomy, Amputation.

\section{INTRODUCTION}

Electricity is omnipresent in our daily lives. Unfortunately, electrical injury can result in some of the most devastating thermal traumas. As opposed to thermal burns, the cutaneous burn size does not correlate with the extent of damage seen in highvoltage (>1000 V) electrical injuries; electrical injuries result in extensive deep tissue injury in addition to various other systemic complications. Morbidities, prolonged hospital stay, multiple visits to the operating room, and long rehabilitation process are not uncommon with these types of injuries.

\section{CASE STUDY}

A 47 year-old Caucasian male, working as an electric company linesman, was transferred to the Montreal General Hospital following a workrelated high-voltage electrical injury (HVEI). While working near high tension power lines, he lost balance and accidently grabbed hold of a wire running $14,000 \mathrm{~V}$ with both hands. He was subsequently

\footnotetext{
*To whom correspondence should be addressed:
}

thrown from the source, fell approximately twenty feet, and suffered blunt head trauma resulting in loss of consciousness.

On arrival to hospital, the patient was alert, oriented and hemodynamically stable. He had 3\% visible total body surface area burns involving bilateral wrists circumferentially and bilateral hands (Fig. 1). This consisted of third degree burns to

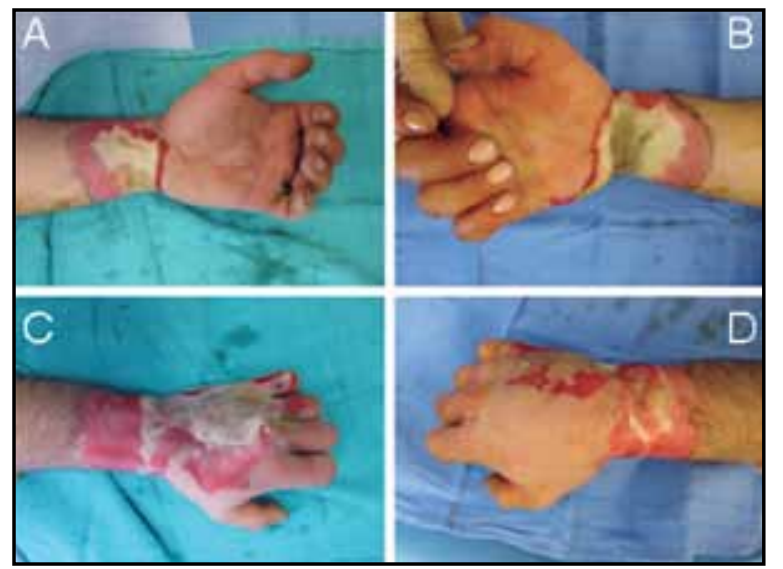

Figure 1: Images on day of admission taken in the operating room. Images A \& B show the volar aspects of the left and right wrists, respectively. Images $C$ \& D show the burns extending onto the dorsal surface of the left and right hand, respectively. 
the volar aspects of both wrists, and second degree burns to the dorsal aspects of both wrists and hands with digits and palms relatively sparred. CT scan of the head revealed a left frontal bone comminuted fracture and a left subdural hematoma with a $3 \mathrm{~mm}$ shift. The patient was immediately taken to the operating room by the neurosurgery team for left frontal craniectomy. In addition, he presented with myoglobinuria and a creatine kinase of 32000 $\mathrm{U} / \mathrm{L}$ and thus copious intravenous hydration was begun.

Within hours of presentation, he was found to have elevated compartment pressures in both hand $(100$ and $70 \mathrm{mmHg}$ on the right and left, respectively) and wrists (91 and $75 \mathrm{mmHg}$ on the right and left, respectively) and was immediately taken to the operating room for bilateral hand and wrist escharotomies and fasciotomies (Fig. 2). Subsequently the patient was taken back to the operating room for serial debridements of necrotic tissue. MRI with gadolinium was performed to delineate the extent of deep tissue injury. Imaging had shown extensive tissue necrosis extending from the distal third of the forearm to the level of the wrists bilaterally, involving both extensor and flexor compartments (Fig. 3).

Despite escharotomies, fasciotomies and serial debridements the patient's left hand became progressively congested with poor doppler signals and low $\mathrm{O} 2$ saturation within each digit. On postburn day 16,9 days since the last debridement, the hand became cold and did not return blood on pinprick in any of the digits. Later that night, the patient was taken to the operating room for ampu-

Figure 2: Post-fasciotomy images of the patient in the operating room. Images A \& B showing wrist fasciotomy, thenar incisions, and carpal tunnel release of left and right hands and wrists, respectively. Images $C$ \& D show fasciotomy incisions on the dorsal aspect of left and right wrists, respectively, as well as the longitudinal metacarpal incisions of the hands.

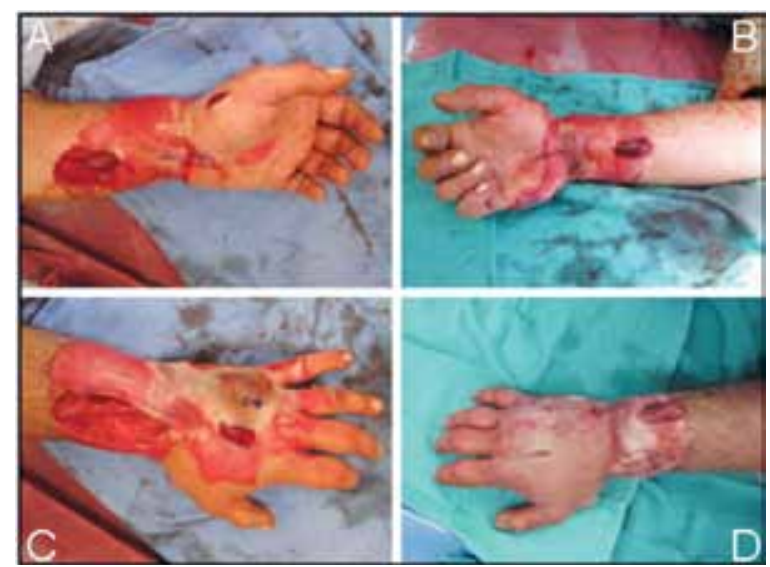

tation of the left hand. After amputation, the patient was left with significant soft tissue defects to bilateral wrists and hands. Bilateral pedicle groin flaps were used for coverage of the defects as well as to increase vascularity to the region (Fig. 4a). Fourteen to twenty-one days after inset of the flaps, the flaps were ready to be separated; final results are shown in Figure $4 \mathrm{~b}$ and $4 \mathrm{c}$.

In summary, the patient remained in hospital for a total of 81 days, 65 of which were spent in the ICU. He was taken to the operating room a total of 7 times for escharotomies, fasciotomies, and serial debridements of bilateral hands and wrists, as well as amputation of his left hand and the necessity of tissue transfers, in the form of pedicle groin flaps, for soft tissue coverage. His course was complicated by rhabdomyolysis with a creatine kinase level peaking at $52235 \mathrm{U} / \mathrm{L}$, which was successfully managed with copious intravenous hydration and diuresis. The patient is currently rehabilitation center, working extensively with physiotherapy for elbow and shoulder mobility. Future plans include extensive tendon transfers to the right hand for improved function.

\section{DISCUSSION}

It is reported that electrical burns constitute between $0.04 \%$ to as high as $32.2 \%$ of admissions to major burn centers $(1,3-6)$. Mortality rates are significant with these types of injuries, reported in the literature to be as high as $59 \%$ (1); the most common cause being secondary to an acute arrhythmia at the scene of the injury(2). They account for approximately 1000 deaths each year in

Figure 3: MRI with gadolinium of left wrist and forearm. The image of left forearm demonstrates the extensive non-enhancing muscles from the distal third of the forearm to the level of the wrist suspicious for myonecrosis involving both extensor and flexor muscles.

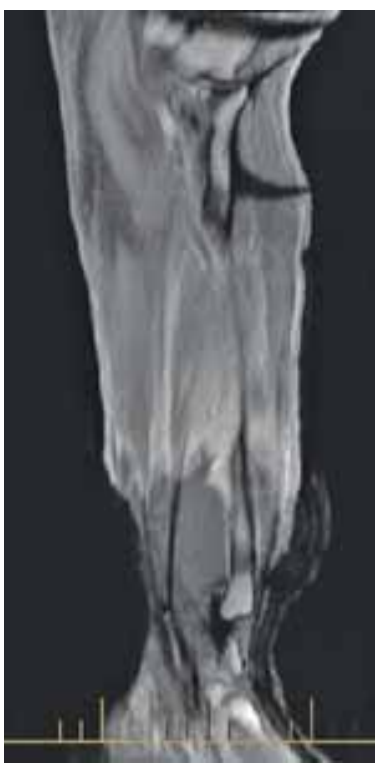




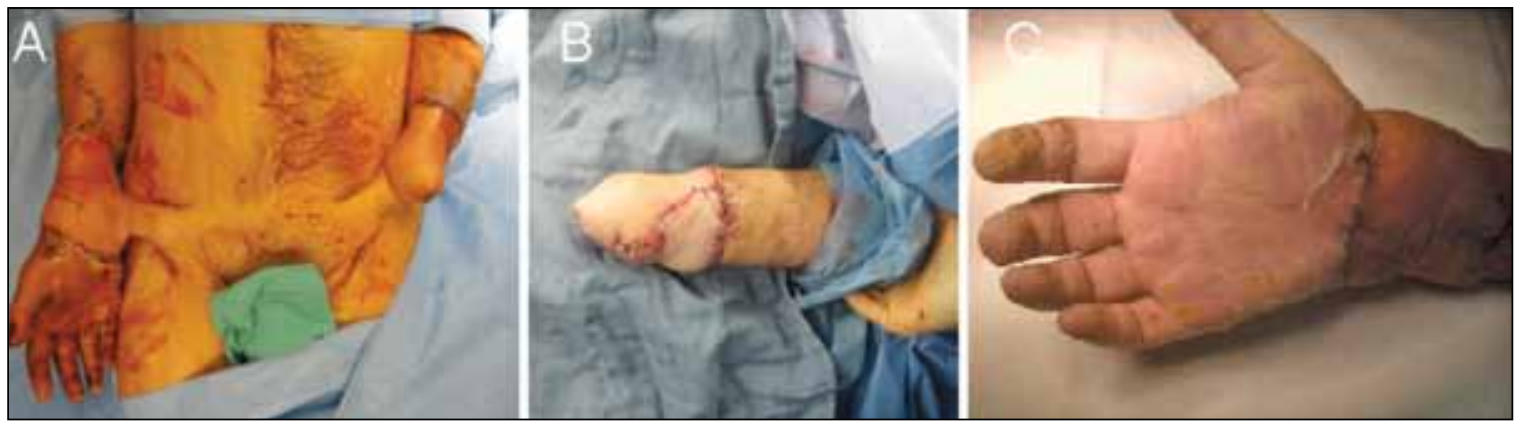

Figure 4: Pre and post separation of pedicle groin flap to bilateral wrists. Image A shows the patient with bilateral pedicle groin flaps attached to bilateral wrists. Image B shows the amputated left hand once flap was separated and inset. Image $\mathrm{C}$ shows the right hand after flap separation.

the United States alone, ranking 5th as the most common cause of occupational deaths (3). Electrical burns are most commonly seen in work related injuries and involve mainly young males. Electrical company linesman, electricians and construction workers are especially at risk $(4,5)$. Sadly, it has been reported that as little as $5.4 \%$ of patients suffering a HVEI are able to return to their previous line of work (2). This can likely be attributed to the fact that these injuries commonly involve the hands and upper extremity $(4,5)$.

Contrary to thermal burns, high-voltage injuries result in extensive deep tissue damage, extending far beyond what can be predicted by TBSA involved, and resulting in higher rate of complications (6). In a case-matched controlled analysis comparing HVEI and thermal burns, Handschin et al. (6) found a significantly higher rate of escharotomy/fasciotomy ( $47 \%$ vs. $21 \%)$, amputations $(19.1 \%$ vs. $1.5 \%$ ), as well as total hospital length of stay (44 days vs. 31 days) in HVEI versus thermal burns.

Commonly seen with HVEI are associated acute injuries which include fractures, head trauma, intra-abdominal injury, renal injury, soft-tissue injury, and acute ocular injury. Associated injuries have been described to occur in $25.1 \%$ of $\operatorname{HVEI}(5,8)$. In addition to associated trauma, patients suffering HVEI are prone to various complications as well. In a study involving 202 electrical injuries, neurologic complications, deep muscle involvement, and amputation of extremities were found to be the most prevalent (4). Similarly, Arnoldo et al. found amputation of extremities or digits and muscle necrosis to be some of the most frequently observed complications, observed in 95 and 68 of the 263 cases of HVEI, respectively (5). As presented in our case, myoglobinuria and fasciotomy are also commonly encountered complications. In the current literature, escharotomy/fasciotomy rates have been de- scribed to be as high as $54 \%$ (7) and amputation rates as high as $49.4 \%$ (2).

Interestingly, Cancio et al. found myoglobinuria to be a factor associated with the need for fasciotomy. Furthermore, they had found myoglobinuria and the need for fasciotomy to be independent risk factors for amputation. Using an equation derived to predict the probability of amputation (8), the patient from our case had a $94.98 \%$ probability of requiring an amputation.

HVEI are devastating injuries associated with a vast array of serious and inevitable complications. The prognosis for these patients depends on the degree of the initial insult as well as the severity of any subsequent complications. Early intervention is the goal with resuscitation and aggressive surgery being the mainstay of management. Considering that most HVEI are work-related, the best way to decrease the morbidity and mortality related to these injuries is prevention. Through public education and work safety programs, most electrical injuries can be avoided. 


\section{REFERNCES}

1. Haberal, M. Electrical burns: a five-year experience-1985. J Trauma. 1986; 26: 103-109.

2. Hussman J, Kucan JO, Russel RC, et al. Electrical injuries morbidity, outcome and treatment rationale. Burns. 1995; 21: 530-535.

3. Martinez JA, Nguyen T. Electrical Injuries. South Med J. 2000; 93: 1165-1168.

4. Maghsoudi H, Adyani Y., Ahmadian N. Electrical and Lightning Injuries. J Burn Care Res. 2007; 28: 255-261.

5. Arnoldo BD et al. Electrical Injuries: A 20-Year Review. J Burn Care Rehabil. 2004; 25: 479-484.
6. Handschin AE et al. A Case-Matched Controlled Study on High-Voltage Electrical Injuries vs Thermal Burns. J Burn Care Res. 2009; 30: 400-407.

7. Garcia-Sanchez V, Gomez-Morell P. Electrical burns: high and low tension injuries. Burns. 1999; 25: 357-360.

8. Cancio LC et al. One Hundred Ninety-Five Cases of HighVoltage Electrical Injury. J Burn Care Rehabil. 2005; 26: 331-340.

9. Koumbourlis AC. Electrical injuries. Crit Care Med. 2002; 30: S424-S430.

James Lee (B.Eng, M.D.,C.M.) completed his Bachelor's degree in electrical engineering and his medical school at McGill University. He will be joining the Plastic Surgery residency program at the McGill University Health Center from July 2010.

Hani Sinno (B.Sc., M.Eng., M.D.,C.M.) is in his forth year of plastic surgery residency at McGill University. He has completed his Bachelor of Science at McGill University Majoring in Physiology. He also completed a Master's of Engineering with Honors at McGill University where his interest focused on Biomedical Engineering and its application to wound healing and reconstructive surgery. He was a junior editor and then a senior editor for the McGill Journal of Medicine during his medical training at McGill University Medical School. His research interests include reconstructive surgery, and biomedical engineering.

Anthony Perkins (M.D., C.M.) completed his medical school at McGill University. He then went on to join the Plastic Surgery residency program at the McGill University Health Center in July 2006.

Youssef Tahiri (M.D., C.M) completed his medical school at McGill University. He then went on to join the Plastic Surgery residency program at the McGill University Health Center in July 2008. He is also currently doing a Masters Degree in Experimental Surgery and is conducting research on Craniofacial Deformities.

Mario Luc (M.D, M.Sc.) obtained his M.D. and M.Sc. from Laval University in 2003 and 2004 , respectively. He then successfully completed a residency in Plastic and Reconstructive Surgery at McGill University in 2008. Following his residency, he went on to complete a combined Hand and Microsurgery Fellowship at the Medical College of Wisconsin. Dr. Mario Luc has joined the McGill University Division of Plastic Surgery in 2009 with a focus on reconstructive hand and microsurgery. 